\title{
EFEITO DA TEMPERATURA E VELOCIDADE DO AR SOBRE A TAXA DE SECAGEM DA MADEIRA DE Pinus elliottii Engelm.
}

\section{EFFECT OF DRYING TEMPERATURE AND AIR VELOCITY ON DRYING RATE OF SLASH PINE LUMBER}

\author{
Elio José Santini ${ }^{1}$ Clóvis Roberto Haselein ${ }^{2}$
}

\section{RESUMO}

Para avaliar o efeito da temperatura e velocidade do ar sobre a taxa de secagem, pe ças de madeira de Pinus elliottii de $25 \times 125 \times 750 \mathrm{~mm}$ foram submetidas à secagem em estufa semi-industrial de convecção forçada. O processo foi conduzido para duas temperaturas e duas velocidades de ar e controlado por meio de um sistema computadorizado. Os resultados mostraram que a taxa de secagem tem uma rela ção diretamente proporcional com a temperatura, velocidade do ar e umidade da madeira. Por meio da an álise de regressão múltipla detectou-se efeito estatisticamente significativo da temperatura e da velocidade do ar sobre a taxa e o tempo de secagem, com um nível de confiança de $99 \%$. Como a importância da velocidade do ar na secagem decresce com a diminuição do teor de umidade, sugere-se, por razões de economia, mais investigações acerca das relações entre as duas variáveis durante o período de taxa de secagem decrescente.

Palavras-chave: taxa de secagem, umidade da madeira, velocidade do ar, temperatura de secagem.

\begin{abstract}
To evaluate the effect of temperature and air velocity on drying rate, slash pine wood pieces of $25 \mathrm{x}$ $125 \times 750 \mathrm{~mm}$ were dried in semi-industrial kiln with forced convection. The process was conduct for two temperatures and two air velocities and controlled by a dat a acquisition system. The results showed that the drying rate has a direct relationship to temperature, air velocity and wood moisture content. Through multiple regression analysis it was possible to detect statistically significant effect of temperature and air velocity on drying rate and drying time, at $99 \%$ confidence level. Since the influence of air velocity on wood drying decreases with moisture content, because of economic reasons, more investigations were suggested about the relationship between the two variables during falling-rate drying period.
\end{abstract}

Key words: drying rate, wood moisture content, air velocity, drying temperature.

\section{INTRODUÇÃO}

A taxa de secagem é uma variável que fornece informações importantes sobre o comportamento da madeira em secagem. A saída mais ou menos rápida da água por unidade de tempo é influenciada por variáveis relacionadas ao material, como estrutura anatômica, e da estufa, como largura da pilha, espessura dos separadores, velocidade do ar, temperatura da madeira e umidade rel ativa do ar (Herzberg et al., 1985). Para Schafer (1973a), contudo, além dos dois últimos fatores, a taxa com que a umidade se movimenta na madeira depende também do gradiente de umidade.

A taxa de secagem é influenciada também pelo método empregado para remover a água da madeira. A principal diferença é que, no método de baixa temperatura o movimento da umidade do centro para a periferia, é um fenômeno de difusão, ao passo que, no de alta temperatura, ocorre um fluxo de massa ou hidrodinâmico (Schafer, 1973b; Vermaas, 1987). A secagem a alta temperatura envolve três fases distintas (Hann, 1964) e apresenta um comportamento diferenciado dos demais métodos. Além disso, a taxa de evaporação nos primeiros estágios da secagem decresce relativamente menos que aquela observada na baixa temperatura, causando uma equaliza ção da taxa de secagem (Schneider, 1980).

Estudos experimentais conduzidos com madeiras de coníferas indicaram que o tempo de secagem pode ser reduzido pelo uso de temperaturas mais altas, ou pelo aumento da velocidade do ar (Herzberg et al.,

1. Engenheiro Florestal, Dr., Professor Adjunto do Departamento de Ciências Florestais, Centro de Ciências Rurais, Universidade Federal de Santa Maria, CEP 97105-900, Santa Maria (RS). santinie@ccr.ufsm.br

2. Engenheiro Florestal, PhD., Professor Adjunto do Departamento de Ciências Florestais, Centro de Ciências Rurais, Universidade Federal de Santa Maria, CEP 97105-900, Santa Maria (RS). haseleic@ccr.ufsm.br

Recebido para publicação em 23/11/2001 e aceito em 18/10/2002. 
1985; Price e Koch, 1981). O aumento linear da taxa de secagem, em razão da elevação da temperatura entre 60 e $180^{\circ} \mathrm{C}$, foi observado em estudos anteriores conduzidos em estufa de laboratório (Santini, 1980). O emprego de altas temperaturas e altas velocidades de ar, nas estufas de secagem, resulta em aumento da taxa de transferência de calor para a madeira e, conseqüentemente, em maiores taxas de secagem.

$\mathrm{O}$ efeito da velocidade do ar sobre a taxa de secagem é mais pronunciado durante o período de taxa constante, quando a madeira possui altos teores de umidade ( Kollmann e Schneider, 1960). Em razão disso, a ocorrência de altas taxas de secagem é comum no início do processo (Schneider, 1972); porém, a partir do momento em que a água superficial é evaporada da madeira, a taxa torna-se decrescente. Durante o período de taxa de secagem decrescente, o efeito diminui continuamente e, eventualmente, torna-se insignificante (Salamon e Mcintyre, 1969).

O teor crítico de umidade em que a velocidade do ar deixa de ser importante varia com a espécie, sendo relativamente baixo para madeiras de pequena espessura, para condições de secagem suaves e para teores de umidade iniciais baixos (Kollmann e Schneider, 1960). Na secagem c onvencional de Liriodendron tulipifera L., Steinhagen (1974) detectou influência significante da velocidade do ar sobre a taxa de secagem apenas até, aproximadamente, $40 \%$ de umidade. Abaixo desse ponto, por razões econômicas, recomendou o uso de velocidades inferiores a $1,25 \mathrm{~m} / \mathrm{s}$ até o final da secagem.

Segundo Milota e Tschernitz (1990), no início da secagem, quando a madeira possui alto teor de umidade, a transferência de calor é o mecanismo controlador e a taxa de secagem estimada é uma função da velocidade do ar e da depressão do bulbo úmido. Quando a madeira está mais seca, a taxa de secagem estimada é uma função da temperatura do bulbo seco e do teor de umidade.

O presente trabalho foi realizado com o objetivo de avaliar o efeito da temperatura e da velocidade do ar sobre a taxa de secagem, calculada pela relação entre a percentagem de água evaporada e o tempo decorrido durante a secagem da madeira serrada de Pinus elliottii.

\section{MATERIAL E MÉTODOS}

O material utilizado no presente estudo é proveniente de árvores de Pinus elliottii Engelm. com, aproximadamente, 26 anos de idade. Toras de $2,5 \mathrm{~m}$ de comprimento foram desdobradas em tábuas de 2,5 $\mathrm{x}$ $12,5 \mathrm{~cm}$ de espessura e largura respectivamente.

De cada tábua, confeccionaram-se três peças de $0,75 \mathrm{~m}$ de comprimento que constituem as amostras de secagem. No momento do corte, retiraram-se dos extremos das amostras seções com 2,5 cm de largura para determinação do teor de umidade inicial da madeira (TUi). A média aritmética entre os teores de umidade de duas seções retiradas dos extremos de cada amostra foi considerada como sendo o TUi desta.

Após sua confecção, obteve-se o peso úmido inicial das amostras de secagem (Pi) com o uso de uma balança mecânica de precisão decimal. A somatória dos Pi de cada amostra e a média de teor de umidade entre as peças componentes da pilha foram consideradas, respectivamente, como sendo o Pi e o TUi da carga de madeira. Essas duas variáveis, Pi e TUi são indispensáveis para o cálculo do TU da carga de madeira durante a secagem em estufa, o qual foi determinado periodicamente pelo método de pesagem da carga, utilizando-se uma célula de carga tipo tração.

Os procedimentos usados para preparação das amostras de secagem e determinação do teor de umidade da madeira seguiram as recomendações de Rasmussen (1961), Simpson (1991) e Mackay e Oliveira (1989).

O gerenciamento do processo de secagem foi realizado por meio de um sistema computadorizado composto de um microcomputador com software específico, unidade de controle e aquisição de dados e estufa de secagem.

A secagem da madeira foi conduzida numa estufa com capacidade para, aproximadamente, $1,0 \mathrm{~m}^{3} \mathrm{de}$ madeira serrada, aquecida por trocador de calor e dotada de circulação forçada de ar. As condições internas da estufa, regulada por meio dos termômetros de bulbo seco e bulbo úmido, caldeira elétrica e aberturas de ventilação, foram comandadas pelo sistema automatizado previamente mencionado. A velocidade de circulação do ar através da pilha foi proporcionada por um ventilador axial capaz de produzir um volume de 
$\underline{\text { Efeito da temperatura e velocidade do ar sobre a taxa de secagem da madeira de Pinus elliottii Engelm. } 101}$

ar de $1,0 \mathrm{~m}^{3} / \mathrm{s}$.

Para cada uma das temperaturas de 50 e $110^{\circ} \mathrm{C}$, quatro cargas de madeira foram submetidas à secagem, sendo duas para cada velocidade de ar utilizada, num total de oito cargas de madeira. A velocidade do ar foi obtida por meio da relação entre o volume de ar produzido pelo ventilador e a área de passagem de ar na pilha. Para as condições normais de fluxo de ar, verificou-se uma velocidade de $2,3 \mathrm{~m} / \mathrm{s}$, ao passo que para obter o valor de $5,0 \mathrm{~m} / \mathrm{s}$, foi necessário interpor um anteparo na frente da pilha de madeira, de modo a reduzir a área de passagem do ar. Esses valores foram confirmados por meio de um anemômetro portátil digital, medindo-se a velocidade no lado de saída do ar na pilha de madeira, nos espaços conferidos pelos sarrafos separadores.

Os programas de secagem foram elaborados para dois métodos distintos: um de baixa $\left(50^{\circ} \mathrm{C}\right)$ e outro de alta temperatura $\left(110^{\circ} \mathrm{C}\right)$, os quais são apresentados na Tabela 1.

A taxa de secagem foi calculada por meio da relação entre a percentagem de água evaporada da madeira e o tempo decorrido, expressa em \%/hora.

TABELA 1: Programas de secagem utilizados para as temperaturas de $50^{\circ} \mathrm{C}$ e $110^{\circ} \mathrm{C}$.

TABLE 1: Drying schedules used for the temperatures of 50 and $110^{\circ} \mathrm{C}$.

\begin{tabular}{r|c|cc|c|c|c}
\hline $\mathrm{TU}(\%)$ & $\mathrm{TBS}\left({ }^{\circ} \mathrm{C}\right)$ & $\mathrm{TBU}\left({ }^{\circ} \mathrm{C}\right)$ & $\mathrm{UE}(\%)$ & $\mathrm{TBS}\left({ }^{\circ} \mathrm{C}\right)$ & $\mathrm{TBU}\left({ }^{\circ} \mathrm{C}\right)$ & $\mathrm{UE}(\%)$ \\
\hline$>70$ & & 47 & 15,8 & & 99,4 & 7,0 \\
$70-50$ & & 46 & 14,0 & & 97,7 & 6,3 \\
$50-40$ & & 43 & 10,5 & & 94,4 & 5,4 \\
$40-30$ & 50 & 40 & 8,3 & 110 & 87,8 & 4,0 \\
$30-20$ & & 35 & 6,2 & & 82,2 & 3,3 \\
$20-15$ & & 30 & 4,4 & & 76,7 & 2,6 \\
$15-10$ & & 28 & 3,8 & & 71,1 & 2,1 \\
\hline
\end{tabular}

Em que: $\mathrm{TU}$ = teor de umidade da madeira; TBS: temperatura do bulbo seco; $\mathrm{TBU}=$ temperatura do bulbo úmido; $\mathrm{UE}=$ umidade de equilíbrio da madeira.

\section{RESULTADOS E DISCUSSÃO}

\section{Tempo de secagem}

As curvas de secagem, elaboradas para a madeira de Pinus elliottii nas duas temperaturas e velocidades de ar, são apresentadas na Figura 1. Uma análise comparativa visual destas evidencia a influência da temperatura e da velocidade do ar no processo. Ao se estabelecer o cálculo de um coeficiente para comparar os dois programas com referência à velocidade do ar, dividindo-se o tempo de secagem na velocidade de $2,3 \mathrm{~m} / \mathrm{s}$ pelo tempo na velocidade de $5,0 \mathrm{~m} / \mathrm{s}$, encontrou-se 1,2 e 1,9 respectivamente para

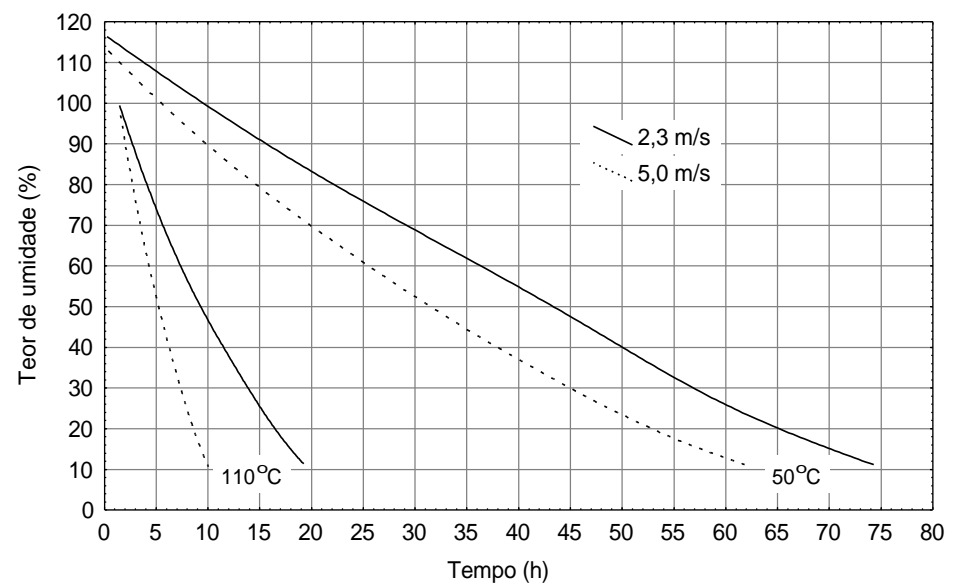

FIGURA 1: Curvas de secagem elaboradas para duas temperaturas de secagem e duas velocidades de ar.

FIGURE 1: Drying curves built for two drying temperatures and two air velocities. 
as temperaturas de $50^{\circ} \mathrm{C}$ e $110^{\circ} \mathrm{C}$. Esses resultados indicam que o efeito da velocidade do ar é quase-insignificante a baixa temperatura, mas a $110^{\circ} \mathrm{C}$ o aumento da velocidade de ar de 2,3 para $5,0 \mathrm{~m} / \mathrm{s}$ praticamente reduziu para menos da metade o tempo de secagem.

Informações gerais sobre as operações de secagem das oito cargas de madeira, como número de observações (leituras), teor de umidade inicial (TUi) e final (TUf), e tempo de secagem, são apresentadas na Tabela 2. Os valores de teor de umidade inicial e final referem-se ao momento em que a estufa atingiu a temperatura programada e quando o software encerrou o processo respectivamente. Após a finalização da secagem, o teor médio de umidade determinado pelo método gravimétrico ficou muito próximo daquele apresentado na Tabela 2. O número de observações representa a quantidade de dados coletados durante o período de secagem, quando foram realizadas entre 40 e 50 leituras por hora. O tempo de secagem refere-se ao período decorrido desde o acionamento da estufa até seu desligamento, quando o teor de umidade final foi atingido.

TABELA 2: Número de observações, teor de umidade e tempo de secagem para as duas temperaturas e velocidades de ar.

TABLE 2: Number of observations, moisture content and drying time for two temperatures and air velocities.

\begin{tabular}{ccc|c|c|c}
\hline TS $\left({ }^{\circ} \mathrm{C}\right)$ & $\mathrm{V}(\mathrm{m} / \mathrm{s})$ & $\mathrm{N}$ & TUI $(\%)$ & TUF $(\%)$ & Tempo $(\mathrm{h})$ \\
\hline \multirow{3}{*}{50} & 2,3 & 3058 & 114,8 & 10,0 & 73,50 \\
& & 3098 & 113,7 & 9,5 & 74,74 \\
& 5,0 & 3194 & 115,2 & 10,5 & 63,00 \\
& & 3089 & 112,5 & 9,5 & 62,21 \\
\hline \multirow{3}{*}{110} & 2,3 & 852 & 101,6 & 9,0 & 19,50 \\
& \multirow{2}{*}{5,0} & 814 & 104,0 & 11,0 & 18,70 \\
& & 446 & 98,0 & 10,5 & 10,07 \\
& & 462 & 95,0 & 9,5 & 11,00 \\
\hline
\end{tabular}

Em que: TS = temperatura de secagem; V = velocidade de ar; $\mathrm{N}=$ número de observações; TUI = teor de umidade inicial; TUF = teor de umidade final.

Para avaliar estatisticamente a influência da temperatura e velocidade do ar sobre o tempo de secagem, procedeu-se à análise de regressão múltipla dos dados, a qual mostrou um bom relacionamento entre as variáveis. O modelo de regressão linear, que melhor se ajustou aos dados, é apresentado na equação $1:$

$\mathrm{t}=126,556-0,892417 \mathrm{~T}-3,71852 \mathrm{~V}$

Em que: $\mathrm{t}=$ tempo de secagem $(\mathrm{h}) ; \mathrm{T}=$ temperatura de secagem $\left({ }^{\circ} \mathrm{C}\right) ; \mathrm{V}=$ velocidade do $\mathrm{ar}(\mathrm{m} / \mathrm{s})$.

O coeficiente de determinação $\left(R^{2}{ }_{a j}=99,85 \%\right)$, o erro-padrão da estimativa $\left(S_{y x}=1,11216\right)$ e o valor de $\mathrm{F}$ de 2399,42 obtidos pela análise de regressão a um nível de confiança de $99 \%$ sugerem que houve um bom ajuste do modelo aos dados.

A inclusão dos teores de umidade inicial (TUi) e final (TUf) na análise de regressão múltipla não teve efeito significativo mesmo a $90 \%$ de confiabilidade pelo teste $t$, e assim essas variáveis foram descartadas. Apesar do alto coeficiente de determinação calculado $\left(\mathrm{R}^{2}=99,97\right)$, a análise dos resíduos indica que o modelo não se ajustou bem ao conjunto de dados. Anteriormente, Taylor e Mitchell (1987) já haviam constatado que o TUi, a densidade da madeira e a quantidade de água removida durante a secagem não tinham efeito significativo sobre o tempo de secagem.

A elaboração de programas tipo temperatura-tempo permite processar a secagem sem a necessidade de se medir periodicamente o teor de umidade da carga de madeira. Para analisar a viabilidade dessa alternativa, foram ajustados modelos matemáticos para as duas velocidades de ar testadas com a temperatura de $110^{\circ} \mathrm{C}$ por meio da análise de regressão com as variáveis teor de umidade e tempo. Optou-se pela alta temperatura, porque o tempo de secagem foi menor.

Dentre os modelos testados, o que melhor se ajustou aos dados é apresentado na equação 2: 


$$
\sqrt{T U}=b_{0}-b_{1} t-b_{2} e^{t}
$$

Em que: $\mathrm{TU}=$ teor de umidade da madeira $(\%) ; \mathrm{b}_{0}, \mathrm{~b}_{1}, \mathrm{~b}_{2}=$ parâmetros estatísticos; $\mathrm{t}=$ tempo de secagem (h); e = base do logaritmo neperiano.

A análise de regressão múltipla realizada para as duas velocidades de ar evidenciou que as equa ções utilizadas para estimar o teor de umidade ajustaram-se bem aos dados, como comprovam o baixo erro-padrão da estimativa, os altos coeficiente de determinação e valor de $\mathrm{F}$ apresentados na Tabela 3 bem como a análise dos resíduos. Além disso, todos os coeficientes estimados, para as equações ajustadas nas velocidades de ar de 2,3 e 5,0 m/s, foram significativos para probabilidades superiores a 99,91\%.

TABELA 3: Coeficientes e estimativas dos parâmetros da análise de regressão entre teor de umidade da madeira e tempo de secagem para a temperatura de $110^{\circ} \mathrm{C}$.

TABLE 3: Regression estimated coefficients and parameters for moisture content and drying time for $110^{\circ} \mathrm{C}$ temperature.

\begin{tabular}{c|c|c|c|c|c|c}
\hline $\begin{array}{c}\text { Velocidade de Ar } \\
(\mathrm{m} / \mathrm{s})\end{array}$ & \multicolumn{3}{|c|}{ Coeficientes } & $\mathrm{R}_{\text {aj }}(\%)$ & $\mathrm{S}_{\mathrm{yx}}$ & $\mathrm{F}$ \\
\cline { 2 - 4 } & $\mathrm{b}_{0}$ & $\mathrm{~b}_{1}$ & $\mathrm{~b}_{2}$ & & & \\
\hline 2,3 & 10,4290 & $-0,359786$ & $-7,57103 \mathrm{E}-10$ & 99,8 & 0,0848 & $176255^{* *}$ \\
5,0 & 10,9887 & $-0,748229$ & $-1,07766 \mathrm{E}-5$ & 99,9 & 0,0543 & $266577^{* *}$ \\
\hline
\end{tabular}

Em que: $b_{0}, b_{1}, b_{2}=$ coeficientes da equação; $R_{a j}^{2}=$ coeficiente de determinação ajustado; $S_{y x}=$ erro-padrão da estimativa; $\mathrm{F}=\mathrm{F}$ de Fisher calculado; $* *$ Significativo a $1 \%$ de probabilidade de erro.

Com base nas informações mostradas na Tabela 4, mantendo-se as condições da estufa nos intervalos de umidade especificados no programa de secagem, a madeira pode ser considerada seca depois de decorridas 19,33h e 10,08h, respectivamente, para as velocidades do ar de 2,3 m/s e 5,0 m/s. Embora a inclusão do teor de umidade inicial não tenha dado uma resposta estatística significativa, sua incorporação ao modelo deve ser considerada, como sugerem as equações de Simpson e Sagoe (1991). No presente estudo, os períodos de tempo relativamente longos no primeiro estágio de secagem (desde verde até $70 \%$ ), verificados para as duas velocidades de ar, são provavelmente em conseqüência dos altos TUi da madeira informados na Tabela 2.

TABELA 4: Programa elaborado com base na equação de regressão ajustada para a temperatura de $110^{\circ} \mathrm{C} \mathrm{e}$ duas velocidades de ar.

TABLE 4: Drying schedules built using the adjusted regression equation for the $110^{\circ} \mathrm{C}$ and two air velocities.

\begin{tabular}{c|c|c|c}
\hline Teor de & Umidade de & \multicolumn{2}{|c}{ Tempo Decorrido (h) } \\
\cline { 3 - 4 } Umidade (\%) & Equilíbrio (\%) & Velocidade de ar 2,3 m/s & Velocidade de ar 5,0 m/s \\
\hline$>70$ & 7,0 & 5,72 & 3,49 \\
$70-50$ & 6,3 & 3,62 & 1,74 \\
$50-40$ & 5,4 & 2,07 & 1,01 \\
$40-30$ & 4,0 & 2,35 & 1,12 \\
$30-20$ & 3,3 & 2,76 & 1,27 \\
$20-15$ & 2,6 & 1,55 & 0,71 \\
$15-10$ & 2,1 & 1,26 & 0,74 \\
Total & - & 19,33 & 10,08 \\
\hline
\end{tabular}

Taxa de secagem

A variação da taxa de secagem da madeira em função da diminuição do seu teor de umidade é ilustrada na Figura 2, para as temperaturas de $50^{\circ} \mathrm{C}$ (A) e $110^{\circ} \mathrm{C}$ (B). Pode-se observar que, nas duas temperaturas e velocidades de ar, a taxa de secagem decresce à medida que diminui o teor de umidade da madeira. Entretanto, a diminuição na taxa foi mais suave apenas na operação de secagem conduzida a $50^{\circ} \mathrm{C}$ e velocidade de ar de $2,3 \mathrm{~m} / \mathrm{s}$. Essa pequena amplitude, detectada na taxa durante a secagem, pode ser atribuída à baixa transferência de calor e massa cujos coeficientes são diretamente proporcionais à 
velocidade do ar (Tremblay et al., 2000). Nas demais, tanto na baixa como na alta velocidade de ar, a taxa de secagem apresentou um decréscimo mais acentuado em função do teor de umidade, mostrando uma relação 2,5 vezes maior entre o início e o final do processo.

A análise da Figura 2A permite deduzir que, à medida que decresce o teor de umidade da madeira abaixo de certos limites, diminui também o efeito da velocidade do ar sobre a taxa de secagem. Para a alta temperatura, embora a mesma tendência seja observada (Figura 2B), a taxa de secagem obtida para a velocidade de $5,0 \mathrm{~m} / \mathrm{s}$ representou o dobro daquela encontrada para a velocidade de ar de $2,3 \mathrm{~m} / \mathrm{s}$.
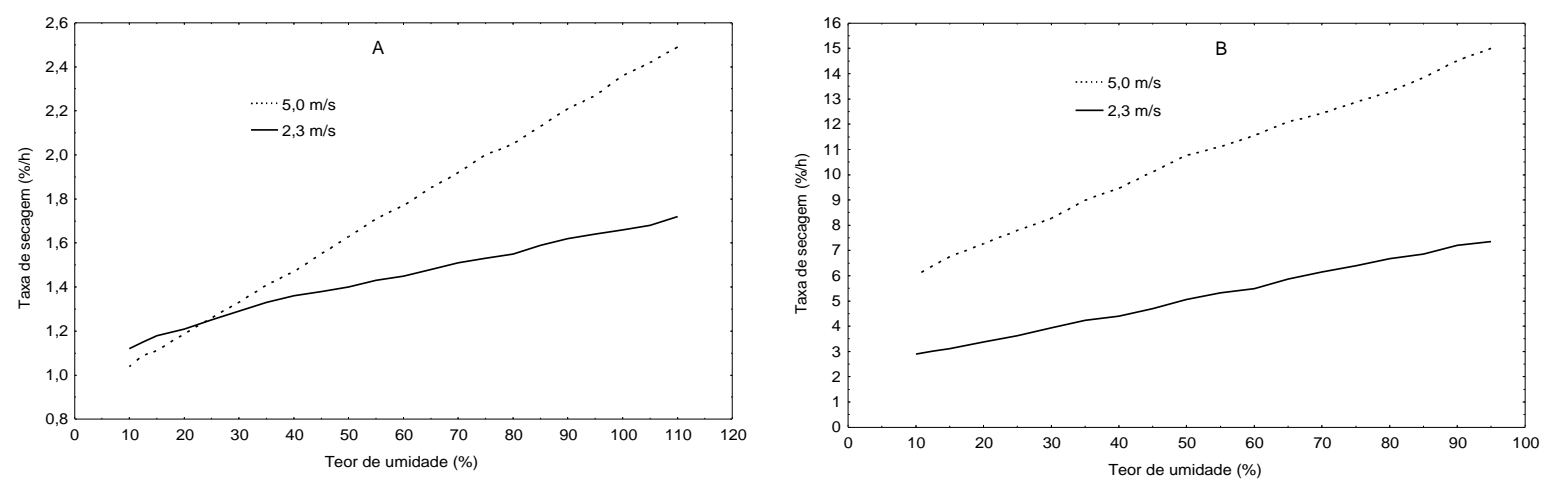

FIGURA 2: Influência da velocidade do ar na taxa de secagem durante a remo ção da umidade da madeira à temperatura de $50^{\circ} \mathrm{C}(\mathrm{A})$ e $110^{\circ} \mathrm{C}(\mathrm{B})$.

FIGURE 2: Effect of air velocity in the drying rate during wood moisture loss at $50^{\circ} \mathrm{C}(\mathrm{A})$ and $110^{\circ} \mathrm{C}(\mathrm{B})$ temperature.

A influência da velocidade do ar sobre a taxa de secagem manifesta-se apenas acima de certos valores de teor de umidade, como o limite de $40 \%$ mencionado por Steinhagen (1974). Esse fato está relacionando provavelmente ao efeito da umidade do ambiente na transferência de calor. Salamon (1969) demonstra que a transferência de calor para a superfície da madeira aumenta à medida que aumenta a umidade do ar. Para os processos, a alta temperatura, contudo, a influ ência da umidade do ar é considerada menos importante (Simpson e Rosen, 1981).

A taxa de secagem diferencial corresponde à razão entre as taxas calculadas para as velocidades de $5,0 \mathrm{~m} / \mathrm{s}$ e $2,3 \mathrm{~m} / \mathrm{s}$. A determinação desse coeficiente e sua distribuição em função da diminuição do teor de umidade da madeira permitiu estabelecer uma comparação visual do efeito da velocidade do ar nos dois processos de secagem, como mostra a Figura 3.

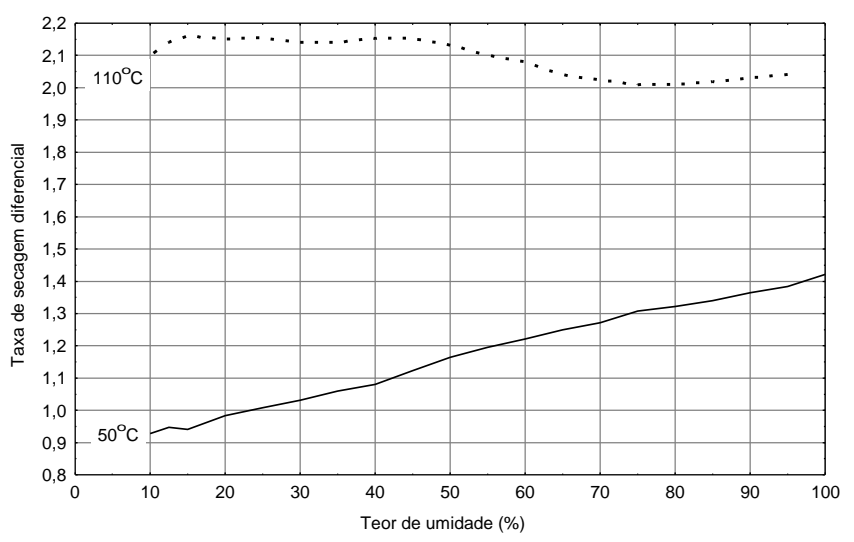

FIGURA 3: Razão entre as taxas de secagem calculadas para as velocidades de ar de 5,0 e 2,3 m/s em função do teor de umidade da madeira, nas temperaturas de 50 e $110^{\circ} \mathrm{C}$.

FIGURE 3: Ratio between drying rates calculated for the 5,0 and $2,3 \mathrm{~m} / \mathrm{s}$ air velocities as a function of wood moisture content, at 50 and $100^{\circ} \mathrm{C}$ temperatures. 
$\underline{\text { Efeito da temperatura e velocidade do ar sobre a taxa de secagem da madeira de Pinus elliottii Engelm. } 105}$

A curva elaborada para a temperatura de $110^{\circ} \mathrm{C}$ mostra que a taxa de secagem obtida na velocidade de ar de $5,0 \mathrm{~m} / \mathrm{s}$ foi aproximadamente o dobro daquela encontrada para $2,3 \mathrm{~m} / \mathrm{s}$, durante praticamente todo o período de secagem. A taxa diferencial manteve-se praticamente inalterada desde o início até o final da secagem. Isto significa que o aumento da velocidade do ar de 2,3 para $5,0 \mathrm{~m} / \mathrm{s}$, não resulta em alterações substanciais na taxa de secagem durante o processo a alta temperatura.

Durante a secagem à temperatura de $50^{\circ} \mathrm{C}$, a Figura 3 mostra que se estabeleceu uma relação decrescente entre as duas taxas de secagem, desde o estado verde at é o teor de umidade de $10 \%$. Entre, aproximadamente, 30 e $10 \%$ de umidade, o valor encontrado foi inferior à unidade, o que indica que, nessa faixa de umidade, a taxa de secagem obtida à alta velocidade de ar foi menor ou, no máximo, igual àquela encontrada para a velocidade mais baixa.

Para avaliar a influência da velocidade do ar e da temperatura interna da estufa sobre a taxa de secagem, aplicaram-se uma análise de regressão múltipla e o modelo de regressão linear que melhor se ajustou aos dados, é representado pela equação 3:

$\ln \mathrm{Tx}=-1,22236+0,0236391 \mathrm{~T}+0,12755 \mathrm{~V}$

Em que: $\operatorname{lnTx}=$ logaritmo natural da taxa de secagem; $\mathrm{T}=$ temperatura de secagem $\left({ }^{\circ} \mathrm{C}\right) ; \mathrm{V}=$ velocidade do ar $(\mathrm{m} / \mathrm{s})$.

O coeficiente de determinação $\left(R_{a j}^{2}=97,63 \%\right)$, o erro-padrão da estimativa $\left(S_{y x}=0,121038\right)$ e o valor de $\mathrm{F}$ de 145,41, obtidos pela análise de regressão a um nível de confiança de $99 \%$, sugerem que houve um bom ajuste do modelo aos dados. Esses resultados indicam que tanto a temperatura como a velocidade de circulação do ar estão intimamente relacionadas com a taxa de secagem. Além disso, os parâmetros estatísticos obtidos pela análise da regressão confirmam que, para o intervalo de temperatura e velocidade de ar estudados, a Equação 3 possibilita estimativas confiáveis de taxa de secagem. Os valores estimados permitem ajustes no programa durante a operação de secagem, visando ao controle do processo e da qualidade da madeira.

\section{CONCLUSÕES}

Os resultados desse trabalho permitem as seguintes conclus ões:

1) $\mathrm{O}$ efeito da velocidade do ar foi mais pronunciado na alta temperatura, em que o uso de $5,0 \mathrm{~m} / \mathrm{s}$ reduziu pela metade o tempo de secagem, em comparação com a velocidade de $2,3 \mathrm{~m} / \mathrm{s}$.

2) Equações de regressão múltipla, que incluem temperatura e velocidade de ar, permitem estimar precisamente o tempo e a taxa de secagem a um nível de confiança de $99 \%$.

3) A taxa de secagem mostrou uma relação diretamente proporcional com a temperatura, velocidade do ar e teor de umidade da madeira. Na temperatura de $110^{\circ} \mathrm{C}$ e velocidade de $5,0 \mathrm{~m} / \mathrm{s}$, a taxa foi praticamente o dobro daquela obtida com $2,3 \mathrm{~m} / \mathrm{s}$, durante todo o período de secagem.

4) A influência da velocidade do ar sobre a taxa de secagem foi decrescente à medida que diminuiu o teor de umidade e se tornou quase desprezível na secagem à baixa temperatura.

\section{REFERÊNCIAS BIBLIOGRÁFICAS}

HANN, R.A. Drying yellow-poplar at temperatures above $100^{\circ}$ C. Forest Prod. J., v. 14, n. 5, p. 215-220, 1964.

HERZBERG, B.L.; TAYLOR, F.W.; ROSEN, H.N. Factors that affect the time required to high-temperature dry pine dimension lumber. Forest Prod. J., v. 35, n. 7/8, p. 34-36, 1985.

KOLLMANN, F.F.P.; SCHNEIDER, A. Der Einfluss der Belüftungsgeschwindigkeit auf die Trocknung von Schnittholz mit Heissluft-Dampf-Gemischen. Holz Roh-Werkstoff, Berlin, v. 13, n. 3, p. 81-94, 1960. For. Abstr., Farnham Royal, v. 21, n. 4, p. 669-670, 1960. Ref. 5085. Resumo.

MACKAY, J.F.G.; OLIVEIRA, L.C. Kiln operator's handbook for Western Canada. Vancouver: Forintek Canada Corp., 1989. 61p. (Special Publication No. SP-31).

MILOTA, M.R.; TSCHERNITZ, J.L. Correlation of loblolly pine drying rates at high temperatures. Wood and Fiber Science, v. 22, n. 3, p. 298-313, 1990. 
PRICE, E.W.; KOCH, P. A note on effects of kiln stick thickness and air velocity on drying time of southern pine 2 by 4 and 2 by 6 lumber. Wood and Fiber, v. 13, n. 2, p. 115-119, 1981.

RASMUSSEN, E.F. Dry kiln operator's manual. Agric. Handb., Madison, n. 188, p. 1-197, 1961.

SALAMON, M. High-temperature drying on its effect on wood properties. Forest Prod. J., Madison, v. 19, n. 3, p. 2734, 1969.

SALAMON, M; MCINTYRE, S. Manipulation of air velocity permits drying time savings. Canadian For. Ind., v. 89, n. 9, p. 49-57, 1969.

SANTINI, E.J. Influência da temperatura na taxa de secagem e contração da madeira de Pinus elliottii proveniente de plantios de rápido crescimento. Série Técnica, FUPEF/UFPR, Curitiba, n. 5, p. 1-15, 1980.

SCHAFER, R.F. What you should know about high-temperature drying. Canadian For. Ind., v. 93, n. 10, p. 70-71, 1973a.

SCHAFER, R.F. Use of time schedules in kiln-drying softwood.Canadian For. Ind., v. 93, n. 11, p. 47-49, 1973 b.

SCHNEIDER, A. Zur Konvektionstrocknung von Schnittholz bei extrem hohen Temperaturen. Erste Mitteilung: Trocknungs-verlauf und Brettemperaturen bei Trocknungstemperaturen von 110 bis $180^{\circ} \mathrm{C}$. Holz Roh-Werkstoff, Berlin, v. 30, n. 10, p. 382-394, 1972.

SCHNEIDER, A. Untersuchungen über den Einflu $\beta$ der Trocknungsbedingungen auf die Gleichmäßigkeit des Verlaufes der Konvektionstrocknung von Schnittholz. Holz als Roh-und Werkstoff, v. 38, n. 3, p. 103-107, 1980.

SIMPSON, W.T. Dry kiln operator's manual. Agric. Handb., Madison, n. 188, p. 1-274, 1991.

SIMPSON, W.T.; ROSEN, H.N. Equilibrium moisture content of wood at high temperatures. Wood Fiber, Lawrence, Kansas, v. 13, n. 3, p. 150-158, 1981.

SIMPSON, W.T.; SAGOE, J.A. Relative drying times of 650 tropical woods: estimation by green moisture content, specific gravity, and green weight density. General Technical Report, Madison, n. 71, p. 1-27, 1991.

STEINHAGEN, H.P. Effect of kiln air velocity, at various moisture content levels, on drying rate of 4/4 yellow-poplar sapwood. Forest Prod. J., v. 24, n. 4, p. 45-47, 1974.

TAYLOR, F.W.; MITCHELL, P.H. Drying pine lumber at very high temperatures and air velocities. Wood Fiber Sci., Madison, v. 19, n. 3, p. 239-245, 1987.

TREMBLAY, C.; CLOUTIER, A.; FORTIN, Y. Experimental determination of the convective heat and mass transfer coefficients for wood drying. Wood Science and Technology, v. 34, n. 3, p. 253-276, 2000. Resumo.

VERMAAS, H.F. Fundamentals of superheated steam and superheated vapour drying. South African Forestry Journal, n. 142, p. 17-23, 1987. 